\title{
Belgeo
}

Revue belge de géographie

$4 \mid 2019$

Les politiques de mobilité urbaine en Europe après la crise : adaptations et innovations

\section{Le service public de covoiturage : vers quelles formes de gouvernance, de régulation et de modèles d'affaires?}

Teddy Delaunay et Nacima Baron

\section{OpenEdition}

\section{Journals}

Édition électronique

URL : https://journals.openedition.org/belgeo/36497

DOI : 10.4000/belgeo.36497

ISSN : 2294-9135

Éditeur :

National Committee of Geography of Belgium, Société Royale Belge de Géographie

\section{Référence électronique}

Teddy Delaunay et Nacima Baron, « Le service public de covoiturage : vers quelles formes de gouvernance, de régulation et de modèles d'affaires? », Belgeo [En ligne], 4 | 2019, mis en ligne le 09 février 2020, consulté le 21 septembre 2021. URL : http://journals.openedition.org/belgeo/36497 DOI : https://doi.org/10.4000/belgeo.36497

Ce document a été généré automatiquement le 21 septembre 2021.

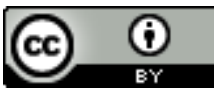

Belgeo est mis à disposition selon les termes de la licence Creative Commons Attribution 4.0 International. 


\title{
Le service public de covoiturage : vers quelles formes de gouvernance, de régulation et de modèles d'affaires?
}

\author{
Teddy Delaunay et Nacima Baron
}

\section{Introduction}

1 En 2019, la nouvelle Loi d'Orientation des Mobilités (LOM) a suscité de vifs débats en France autour du financement des transports collectifs dits « du quotidien ». Cette loi votée fin novembre 2019 s'inscrit dans un double contexte. D'un côté, des contraintes économiques lourdes pèsent sur les transports : insuffisance croissante du Versement Transport ( $\left.\mathrm{VT}^{1}\right)$ pour financer l'investissement et l'exploitation des réseaux de transports collectifs urbains (Richer, 2017); hausse tendancielle du coût des déplacements pour les ménages (Ferret, Demoly, 2019). De l'autre, à l'heure du changement climatique et de la transition énergétique, la loi LOM est marquée par l'affirmation d'une injonction à la durabilité et à la sobriété. Au croisement de ces deux impératifs, le covoiturage s'affirme dans cette loi comme une solution d'action publique écologique et économique pour améliorer l'accessibilité de périphéries métropolitaines à l'urbanisation diffuse.

2 L'intérêt des pouvoirs publics français sur ce sujet n'est toutefois pas nouveau, puisque les acteurs territoriaux sont encouragés à soutenir la pratique du covoiturage depuis le milieu des années 1990, sans véritable succès toutefois. La pratique est en déclin depuis les années 1970 en France (CGDD, 2016), comme d'ailleurs dans de nombreux pays développés (Shaheen, 2018). Cette tendance s'illustre notamment à travers la baisse continue entre 1990 à 2016 du taux d'occupation des véhicules, de 1,78 à 1,58 passagers par véhicule pour tous les motifs de déplacements (CEREMA, 2016; CGDD, 2018). Ainsi aujourd'hui en France, en moyenne, seuls $3 \%$ des déplacements pendulaires sont 
réalisés en covoiturage, dont au moins $50 \%$ dans le cadre familial [pratique d'accompagnement] (ADEME, 2017). La faible diffusion du covoiturage sur courte distance s'explique par les contraintes d'organisation et de perte de temps que la pratique engendre et qui créent une inadéquation profonde entre le covoiturage et la mobilité quotidienne.

3 Toutefois, au-delà de l'identification des freins et des leviers à la pratique, largement documentés (Vincent, 2008; Barthes, 2009 ; Creno, 2016; ADEME, 2017; Bulteau et al., 2019), l'étude de la manière dont s'est structuré le marché, mais aussi de l'encadrement et du soutien accordé par les acteurs territoriaux à ce secteur, offrent également d'autres pistes d'explications pour comprendre cette diffusion contrariée. En France, les services de covoiturage se sont développés selon une logique commerciale et concurrentielle ayant conduit à une atomisation des acteurs et à un morcellement des initiatives, défavorables à la diffusion de la pratique (Vincent, 2008). Par étape, l'investissement des pouvoirs publics sur le sujet s'est progressivement renforcé, à travers la promotion, le développement d'outils incitatifs et le financement de services et d'infrastructures (Delaunay, 2018). Désormais, de nombreux acteurs appellent à aller plus loin dans l'intégration du covoiturage dans l'action publique de mobilité. Ils proposent de sortir les services de covoiturage d'une logique commerciale et de les inscrire dans une logique de Délégation de Services Public (DSP2). Les pouvoirs publics sont à présent incités jouer un véritable rôle d'Autorité Organisatrice de Mobilité à travers la planification, la gestion et le financement des services, mais aussi de plus en plus, à travers l'établissement de monopoles territoriaux pour consolider le marché émergent des opérateurs de covoiturage de courte distance.

4 Cette transition se concrétise dans la LOM et résulte d'un long processus, enclenché il y a plus de vingt-cinq ans. Aussi, afin de rendre compte des enjeux qui ont amené à la situation actuelle, elle-même transitoire et susceptible de scénarios d'évolution pluriels, nous analysons ici le processus d'intégration du covoiturage dans les politiques publiques de mobilité et l'évolution des modèles de gouvernance établis entre les acteurs publics et privés autour de cette solution de déplacement.

5 Pour cela, nous mobilisons un cadre théorique et méthodologique issu de la sociologie de l'innovation. La méthode d'enquête retenue repose sur le recensement des principaux acteurs impliqués dans le covoiturage de proximité et sur la réalisation d'entretiens semi-directifs. Le périmètre de l'étude se restreint au cas français, il ne concerne pas un territoire en particulier car les opérateurs de mobilité enquêtés s'investissent dans différentes régions françaises. Ils peuvent en effet fournir leur service à des territoires, allant de petites communes rurales isolées aux grandes régions métropolitaines. D'ailleurs, le caractère déterritorialisé, tant des services que des politiques de promotion du covoiturage mises en œuvre, explique en partie la faible diffusion de la pratique. Nous verrons ainsi que la volonté des acteurs de pénétrer le champ du service public s'explique aussi par une volonté de s'inscrire davantage dans une logique de territorialisation et d'institutionnalisation.

6 Le plan de l'article est le suivant: après une première partie consacrée au cadre théorique et méthodologique, nous retraçons le processus de développement du covoiturage et les modalités de son intégration dans le champ de l'action publique. Puis, nous précisons comment s'amorce une diversification des formes de gouvernance et de promotion du covoiturage à travers, d'une part, l'intégration des opérateurs de covoiturage dans le champ du service public de transport, et d'autre part, à travers 
l'implication croissante des entreprises historiques de transports collectifs urbains sur ce marché. En fonction du type d'acteurs impliqués, cette transition augure un potentiel renforcement de la place du covoiturage dans les services publics de mobilité, mais peut aussi se traduire par une contention de ce mode dans les marges de l'offre de mobilité. En outre, ces évolutions soulèvent de nombreux enjeux en termes de mise en œuvre et de régulation, et posent également question quant au renforcement de la place de l'automobile dans le système de mobilité.

\section{Méthodologie et cadre théorique}

\section{Le covoiturage : une lecture théorique par la sociologie de l'innovation}

7 L'article mobilise les cadres la sociologie de l'innovation, sachant qu'innovation ne veut pas dire invention récente : le covoiturage, en tant qu'activité sociale, est aussi ancien que l'invention de l'automobile. Les travaux d'Alter (2003) et d'Akrich et al. (1988) expliquent que la diffusion d'une innovation ne dépend pas de la valeur intrinsèque de celle-ci, mais des réseaux d'acteurs sociaux, politiques et économiques qui contribuent ou non à son déploiement. Les innovations font ainsi l'objet de négociations et de controverses afin de traverser différents univers de sens et de s'adapter au contexte territorial et culturel dans lequel elles se diffusent. Pour Desportes (2005), une solution technique dans le transport ne s'impose pas parce qu'elle serait plus performante que les précédentes, mais parce qu'elle fait l'objet d'un processus d'appropriation par des acteurs qui la réinventent et la transforment grâce à un " essaim d'imitateurs " (Alter, 2003). Ces derniers s'emparent de l'innovation, la copient, l'améliorent, lui confèrent des usages spécifiques, ce qui conduit à des " grappes d'innovations » dans les champs institutionnels, culturels, sociaux ou économiques (Alter, 2003). Toutefois, durant cette période d'appropriation, d'autres acteurs peuvent aussi se saisir de l'innovation pour la freiner, la détourner, la cantonner à certains domaines, car l'introduction de la nouvelle solution de transport perturbe un ordre établi, remet en cause des monopoles, des rentes, des positions acquises et nécessite une adaptation de la part de ceux qui ne l'ont pas initiée et/ou souhaitée. Cette lecture des aléas de l'institutionnalisation de l'innovation nous aide à formuler l'hypothèse selon laquelle l'émergence très timide du covoiturage en France, malgré un discours public très positif, est probablement liée à un problème de réception de cet objet, dont l'irruption dans des cercles de négociation autour du futur des transports et des mobilités peut bousculer des intérêts et des positions acquises. Nous proposons donc de développer un questionnement critique sur le niveau d'intérêt partagé, ou au contraire d'antagonisme que peuvent développer les acteurs privés (entre eux) et les acteurs privés et publics à l'égard de la diffusion du covoiturage dans un contexte apparemment porteur sur le plan législatif et politique.

\section{Dispositif d'enquête}

8 La recherche a débuté par le recensement d'acteurs impliqués dans le covoiturage de proximité puis sur l'organisation d'entretiens approfondis avec trois types d'opérateurs : (i) les grands groupes privés qui opèrent pour le compte de collectivités territoriales des services de transport collectifs; (ii) des jeunes entreprises privées du 
secteur digital spécialisées dans l'offre de services de covoiturage; (iii) des responsables de collectivités locales intéressés ou confrontés à ces pratiques. Cinq entretiens semi-directifs approfondis ont été réalisés auprès des directions de l'innovation chez cinq transporteurs (SNCF Transilien, Coordinatrice de Programme; RATP, Responsable stratégie, innovation et développement; RATP Dev, Responsable Marketing; Kéolis, Direction offre et Services Nouvelles Mobilités; Transdev, Responsable Innovation France). Quinze entretiens ont aussi été réalisés auprès de fondateurs de startups du covoiturage (Sharette, Ecov, Rezopouce, Microstop, Voiture \& Co, OuiHop', Cityway, Wayzup, Citygoo, Taxito, PADAM, Karos, Idvroom, Taxistop, La Roue Verte). Une vingtaine d'entretiens ont été menés auprès d'élus et d'agents de collectivités territoriales en Île-de-France (communes, intercommunalités, Conseil régional et départemental, Parc Naturel Régional, Syndicat des Transports d'Ile-deFrance).

9 Le guide d'entretien a été construit avec une série de questions communes à tous les interlocuteurs (niveau de connaissance et type de représentations à l'égard du covoiturage courte distance, actions menées pour le favoriser, obstacles rencontrés, expérimentations réalisées et leurs effets, vision du positionnement et des attentes des autres acteurs du système de mobilité à l'égard du covoiturage). Des questions adaptées au profil de chaque interlocuteur et orientées sur les politiques publiques pour les parties prenantes institutionnelles et sur la dynamique économique du secteur pour les acteurs privés ont aussi été posées. Les propos recueillis ont fait l'objet d'une transcription exhaustive et leur analyse a permis de développer une lecture à la fois historique et stratégique qui souligne d'abord la variabilité des cadres institutionnels et la discontinuité des stratégies de gouvernance de ce champ d'activité.

\section{L'intégration progressive et contrariée du covoiturage dans le champ d'action publique}

\section{Le covoiturage a d'abord été lancé dans la sphère publique à travers l'accompagnement des collectivités aux plans de déplacement d'entreprise}

$10 \mathrm{Au}$ cours des années 1990-2000, plusieurs évolutions législatives occasionnent l'apparition du covoiturage comme objet d'action publique. Tout d'abord, la loi d'orientation des transports intérieurs (LOTI) de 1982 instaure les Autorités Organisatrices de Transport (AOT). Ensuite, la Loi sur l'Air et l'Utilisation Rationnelle de l'Énergie (LAURE) en 1996 oblige les AOT à la mise en place de Plans de Déplacements Urbains (PDU) et des Plans de Déplacements Entreprises (PDE), dont l'objectif est d'engager un ensemble de mesures d'action publique visant à opérer un report modal depuis la voiture individuelle vers les transports collectifs. Dans ces documents, il est donné aux AOT la possibilité d'encourager le covoiturage, au même titre que les transports collectif, afin de lutter contre la dépendance automobile (Dupuy, 1999).

11 Jusqu'au début des années 2010, le principal outil de promotion du covoiturage est le PDE. L'objectif est d'encourager «les entreprises et les collectivités publiques à favoriser le transport de leur personnel, notamment par l'utilisation des transports en commun et du covoiturage » (article 28-6). Le covoiturage n'est cependant pas forcément la pièce 
centrale des PDE, mais la volonté de rationalisation des coûts de stationnement et donc de faire des économies sur le foncier conduit de nombreuses entreprises à enclencher des démarches sur le sujet (Clavel, Legrand, 2009 ; Faessel, 2014 ; Lejoux, 2014).

Les Plans de Déplacement d'Entreprise permettent l'émergence d'un type d'entreprise de covoiturage fondée sur le conseil en management dans le cadre d'un modèle d'affaire dit «Business-to-Business ». L'entreprise engagée dans un PDE paye un forfait annuel pour que l'entreprise de covoiturage sensibilise les salariés et leur fournisse une plateforme internet d'intermédiation de covoiturage dédiée à l'entreprise, permettant à l'offre et à la demande de se rencontrer.

En outre, au tournant des années 2000, des collectivités locales engagent également des politiques transversales de durabilité (en général des documents cadre appelés Agenda 21) organisés en plusieurs volets thématiques. Elles s'intéressent aussi, à ce moment au covoiturage, et selon deux types de dispositifs.

Le premier dispositif est le Plan de Déplacement des Administrations (PDA), assez proche des PDE, qui porte sur la réforme des pratiques de déplacement domicile-travail des employés de la collectivité. Dans ce cas, comme pour les entreprises, les collectivités (et plus largement les administrations) achètent à des prestataires des plateformes internet de mise en relation entre conducteurs et passagers. En outre, les collectivités proposent également dans ce cadre des services d'intermédiation à destination de l'ensemble des résidents du territoire. Les sociétés privées qui accompagnent le déploiement des PDE assurent aussi le marché des PDA : elles gèrent les sites web et prodiguent des conseils en mobilité sous la forme de réunions de sensibilisation.

Le second dispositif consiste en des politiques de planification routière et d'aménagement d'aires de stationnement dédiées au covoiturage. En 2004, les Conseils départementaux reçoivent la compétence d'entretien des routes nationales à la suite de l'acte III de la Décentralisation initié par Jean-Pierre Raffarin. Dans un premier temps, quelques départements pionniers réalisent des aires de covoiturage situées sur de grands axes, à proximité de bretelles de raccordement ou bien lorsque la pratique d'un covoiturage spontané, souvent qualifié de sauvage, est identifiée. Ces aires peuvent regrouper plusieurs dizaines ou seulement quelques places de stationnement. Les aires sont censées à la fois sécuriser la pose et dépose des covoitureurs et des covoiturés et fonctionner comme des parkings sécurisés pour les pratiquants qui laissent leur voiture à la journée. L'aménagement de ces aires intéresse peu à peu d'autres catégories d'acteurs privés : gestionnaires des routes et autoroutes, entreprises de création et de gestion de lieux de stationnement, ce qui participe à la diversification du jeu d'acteurs et des enjeux autour du covoiturage.

\section{Profusion des initiatives et effets pervers des plans de déplacements}

Grâce à la diffusion de Plans de Déplacement d'Entreprise, un marché rentable de prestations s'ouvre alors pour les petites sociétés de covoiturage. Entre 2000 et 2015, environ soixante entreprises de covoiturage de proximité se créent en France (Delaunay, 2018) et entrent en concurrence pour obtenir les différents marchés de plan de mobilité. Des dizaines de sites internet proposant à des publics très peu nombreux, sur des périmètres de transport qui peuvent se chevaucher ou s'emboîter, des trajets 
qui sont eux-mêmes peu nombreux. L'écueil réside dans le fait que le modèle d'affaires des opérateurs de covoiturage n'est pas commercial au sens où il reposerait sur le prélèvement de commission sur chaque trajet réalisé. Le covoiturage courte-distance fonctionne sur le principe de la vente d'un service auprès d'un client (PDA : public PDE : privé) à destination de ses salariés. L'intérêt premier des opérateurs n'est pas qu'un grand nombre d'usagers covoiturent, mais surtout de multiplier les établissements clients. Plus un opérateur vend de plateformes, plus il est rentable et plus il capte de marchés, plus il élargit son périmètre spatial et gagne en visibilité.

Ceci crée une cannibalisation de l'offre sur un même périmètre géographique, puisqu'on peut rencontrer alors une plateforme financée par une collectivité, plusieurs plate-formes financées par des entreprises avec ou sans appui public, plus des plateformes nationales qui opèrent sans restriction territoriale. Ainsi aucune plateforme n'atteint de taille critique qui permettrait de trouver facilement un équipage et faire effet boule de neige.

Ainsi, la vogue du covoiturage courte distance de la décennie 2000 présente des limites : un objet d'action publique mal défini et une gouvernance floue qui conduit à l'investissement d'une trop grande diversité d'acteurs sur le sujet. Le covoiturage est perçu par les acteurs territoriaux comme une solution de mobilité moderne de par son caractère digital, peu onéreuse et rapide à mettre en œuvre, pouvant être investie au titre de compétences et d'objectifs très variés (transport et accessibilité, protection de l'environnement, développement économique, solidarité et inclusion sociale, gestion du trafic automobile). Les collectivités s'en emparent aussi, souvent, dans une optique symbolique ou avec une volonté d'affichage autant que d'efficience. Enfin, certains acteurs territoriaux s'investissent dans l'optique d'affirmer leur présence et leur existence, par exemple les Parcs Naturels Régionaux qui à travers le covoiturage s'emparent des enjeux relatifs à la mobilité. Enfin, les prestations de services des entreprises ne sont pas ou peu territorialisées. Les mêmes plateformes numériques sont dupliquées d'un territoire à l'autre, et contrairement aux autres modes de transports, elles manquent de tangibilité et de visibilité dans l'espace public. Cette absence de tangibilité du covoiturage empêche les acteurs territoriaux de s'approprier ces services qu'ils reconnaissent pour la plupart ne pas bien comprendre. Il est notamment impossible pour les élus locaux de savoir où, quand et à qui la plateforme peut fournir un service. Cette profusion des initiatives, la concurrence entre les opérateurs privés et le caractère déterritorialisé des initiatives va progressivement entraîner un repositionnement des sociétés de covoiturage à l'égard du secteur public.

\section{Recomposition de la gouvernance du covoiturage : diversification des acteurs et tensions entre dérégulation et interventionnisme public}

\section{Les entreprises de covoiturage entre concurrence sauvage, appel à la régulation et statu quo}

Nos entretiens réalisés en 2015 et 2016 auprès de quinze entreprises de covoiturage conduisent à identifier deux types de positionnement de ces acteurs: (i) ceux qui militent pour une libéralisation du secteur; (ii) ceux qui souhaitent intégrer leur 
produit dans l'offre de transport public pour que les autorités subventionnent la pratique encore émergente.

Les startups du premier groupe (i) apparaissent le plus souvent comme résignées, c'està-dire déçues par les difficultés à convaincre les pouvoirs publics de l'intérêt de financer ce type de pratique, et présentent la collaboration avec les pouvoirs publics comme risquée, hasardeuse, volatile, épuisante. Une autre frange se présente comme franchement réfractaire à l'idée de collaborer avec la puissance publique, considérant que les visions et objectifs divergent et que les cadres et modes d'action du monde privé et de la gouvernance locale sont incompatibles. Ces entreprises innovent de manière particulièrement disruptive, selon une logique que l'on peut qualifier " d'ubérisation ». Ce sont des entreprises de taxis non-professionnels qui présentent leur service comme étant du covoiturage, mais qui ne respectent pas la notion de partage de frais de déplacement ${ }^{3}$. Elles tentent de se développer le plus rapidement possible afin d'intéresser des investisseurs pour être revendues à des sociétés plus importantes. Ce type d'opérateur reste à l'écart des acteurs publics, attendant que les pouvoirs publics laissent le marché se structurer dans un cadre libre et concurrentiel avant que des formes de régulation ne se mettent en place et ne les contraignent éventuellement sur le plan réglementaire. Ces acteurs concentrent leurs investissements dans les espaces denses et métropolitains où ils estiment qu'il existe une offre et une demande suffisante et délaissent les territoires peu denses.

21 Un second profil de société (ii) cherche l'ombrelle de l'acteur public et pense pouvoir atteindre les usagers et démocratiser l'usage du covoiturage en passant par la puissance publique pour plusieurs raisons : nécessité et urgence de produire un cadre normatif et de gouvernance clair afin de réduire la concurrence territoriale entre les plateformes, volonté de stimulation de la pratique auprès des ménages et des usagers de transport collectifs à travers un subventionnement de la pratique, attente d'un financement d'une plus grande quantité d'aménagements d'aires de covoiturage et d'infrastructures et d'outils dédiés (voies réservées, système d'information multimodal, intégration tarifaire). Ces entreprises n'attribuent plus seulement aux collectivités un rôle de client, comme lorsqu'elles distribuaient des sites internet d'appariement des équipages dans le cadre des démarches des PDE/PDA. Elles les engagent à jouer pleinement leur rôle d'autorité organisatrice en pilotant et en coordonnant les projets menés sur leur périmètre territorial de compétence.

$\mathrm{Au}$ sein de ce deuxième groupe de sociétés de covoiturage, on trouve deux groupes. Celles qui souhaitent que la puissance publique soit un intermédiaire entre elles et leurs clients finaux et, si possible, qu'elle soit le garant d'un ordre territorial conduisant à la protection de monopoles de marché plus ou moins explicites, de manière à stabiliser le cadre concurrentiel. D'autres proposent des services qui reposent sur la logique de "l'autostop organisé ». Ces dernières veulent déployer sur la voirie des arrêts de covoiturage localisés à des emplacements précis sur le bord de la route, où les usagers peuvent pratiquer l'autostop de manière sécurisée mais surtout en étant assurés que les demandeurs seront rapidement pris en charge par un conducteur ${ }^{4}$. Ces acteurs proposent en définitive des services de covoiturage extrêmement territorialisés, qui requièrent une connaissance fine du territoire afin de planifier leur réseau de points d'arrêts en fonction des flux routiers existants.

Le modèle auquel ces deux types de sociétés adhèrent se rapproche en définitive de celui de la délégation de service public de transport, qui garantit au transporteur le 
monopole sur le territoire de compétence de la collectivité, et qui est financé par l'impôt au nom des externalités positives qu'il produit (Lefevre, Offner, 1990). Depuis la promulgation de la loi MAPTAM (Modernisation de l'Action Publique Territoriale et d'Affirmation des Métropoles) de 2014, l'action des grandes collectivités dans l'organisation des mobilités se renforce et leur offre la possibilité d'investir davantage de ressources dans le covoiturage. Les Autorités Organisatrice de Transport sont devenues des Autorités Organisatrices de Mobilité et peuvent intervenir dans le champ du covoiturage, de l'autopartage et du vélo. Elles peuvent depuis lors utiliser le Versement Transport (VT) pour développer ou encourager la pratique du covoiturage. Elles peuvent a priori utiliser le VT pour rémunérer un conducteur acceptant de covoiturer, à condition que celui-ci ne fasse pas de bénéfice et ne touche pas au final un montant supérieur au barème kilométrique.

Pour parvenir à ces relations privilégiées avec les institutions publiques, ces sociétés sont prêtes à des concessions. Comme ce sont des entreprises du digital, l'un des enjeux majeurs est de partager les données de mobilité, c'est-à-dire à transmettre les informations relatives aux flux des conducteurs. Ces flux, en temps et en volume, permettent alors aux collectivités de monter en puissance sur le plan technique et de renforcer leur capacité à concevoir et mettre en œuvre des documents de planification du covoiturage plus pertinents. Cette stratégie paraît favorable à une rationalisation du marché et à une meilleure visibilité de l'offre.

\section{Enjeux liés à l'établissement d'un monopole territorial du covoiturage}

Toutefois, la mise en place d'un monopole territorial pour les entreprises de covoiturage soulève plusieurs questions. Tout d'abord, la garantie d'un monopole semble difficile à obtenir, puisque par essence les services de covoiturage opèrent sans restriction territoriale. Ce n'est pas parce qu'une collectivité oriente les entreprises de son territoire vers une startup que d'autres startups ne pourront pas opérer sur ce même territoire.

Plus fondamentalement, la manière dont est défini le monopole pose question. D'une part, de multiples formes de services de covoiturage existent, et chacune s'adresse à des publics différents ${ }^{5}$. Il semble donc nécessaire que la collectivité ne se limite pas à garantir le monopole à une seule entreprise de covoiturage, mais qu'elle soit en mesure de diriger les entreprises vers les services qui correspondent le mieux aux objectifs qu'elles poursuivent. Pour y parvenir, une procédure de sélection en amont des startups partenaires pourrait être envisagée, ce qui permettrait à la collectivité d'établir une liste d'acteurs présélectionnés sur lesquels elle détient des éléments précis. Cela pose en définitive la question de la libre concurrence : est-ce que cela doit passer par un appel d'offre pour retenir un opérateur unique, ou bien la rémunération devra-t-elle être accordée aux utilisateurs de toutes les solutions de covoiturage qui respectent les règles établies par la collectivité?

La durée du monopole doit aussi être posée : court ou moyen terme? Au regard du caractère innovant des technologies déployées, des durées courtes seraient certainement à privilégier dans un premier temps. Par ailleurs, des dérives s'observent si la durée du mandat est trop longue. Certaines startups ont obtenu des marchés publics depuis parfois plus d'une dizaine années. Dans ce contexte, elles ont parfois la 
main mise sur la politique de covoiturage du territoire et parviennent à se maintenir en place alors que les bilans de leurs opérations et la qualité de leurs prestations sont questionnables. Le témoignage d'un agent d'une collectivité territoriale permet de comprendre comment une startup opérant pour le compte d'une collectivité tire avantage d'un ancrage local ancien pour se maintenir en place. La collectivité en question n'a pas développé en interne des compétences sur le covoiturage, et c'est le prestataire qui prend en charge la majeure partie de la politique de covoiturage du territoire. Ce dernier détient les données, sélectionne celles qu'il transmet ou non à la puissance publique, pratique une forme de rétention ou de captation de fait du pouvoir, et peut de ce fait influer sur les décisions de l'administration.

Pour éviter ce type de situation, un organisme ayant des fonctions de contrôle et de supervision pourrait être envisagé. Des agences spécialisées, relevant de l'État ou des institutions décentralisées pourraient jouer ce rôle. Des bureaux d'études externes pourraient également évaluer les actions menées par le ou les mandataires. Cela demande toutefois de préciser quels objectifs sont poursuivis, et donc de définir des méthodes standardisées de mesure et d'évaluation des politiques de covoiturage. Si la collectivité travaille avec plusieurs mandataires, elle doit travailler sur la coordination, ce qui peut être lourd en termes organisationnels.

D'autre part, quelle doit-être l'étendue du monopole? Sur tout le territoire ou bien doit-il être divisé en lots? Quid de la concurrence avec les transports collectifs ? La mise en cohérence de ces offres de services suppose que des passerelles entre les acteurs qui les opèrent soient mises en place, ou bien qu'un seul et unique acteur opère les deux types de services.

Sur ce point, il serait aussi intéressant de réfléchir à l'idée de "startups ou d'entreprises publiques de covoiturage ", sous une forme équivalente à celle des Sociétés d'Économie Mixte par exemple, et que soit mise en place une concurrence pour le marché et non plus sur le marché, pour permettre le développement de services de covoiturage ayant des ressources financières conséquentes et surtout qui ne poursuivent pas uniquement un objectif de profits, mais davantage celui de l'intérêt général, défini conjointement entre acteurs privés et publics.

Une autre solution réside dans le fait de ne plus se tourner vers les entreprises de covoiturage, mais de confier la charge d'organiser le covoiturage aux transporteurs qui opèrent dans le cadre de Délégations de Services Publics. Cette solution présente plusieurs intérêts, notamment en termes d'intermodalité et d'intégration des modes de transports, mais cela peut aussi avoir des effets contreproductifs. Il importe de préciser comment les transporteurs se saisissent du covoiturage et quels objectifs ils poursuivent à travers leur investissement sur le sujet.

\section{L'arrivée des grands opérateurs de mobilité sur le marché public du covoiturage courte distance : contention du covoiturage et statu quo}

Avant l'essor du covoiturage longue distance via le service Blablacar, les attentes des collectivités territoriales à l'égard des transporteurs en matière de covoiturage étaient limitées, voire inexistantes. Certaines collectivités se montraient même parfois 
méfiantes à l'égard des transporteurs leur proposant des services de covoiturage, considérant que ce n'est pas leur rôle de proposer ce type de service.

Avec la loi du 12 juillet 1999 "relative au renforcement et à la simplification de la coopération intercommunale» (dite "loi Chevènement»), de nombreuses communautés d'agglomération sont devenues des autorités organisatrices de transport urbain (AOTU). Ces transformations se sont accompagnées, entre 1999 et 2004, d'une extension géographique des périmètres de transport urbain pour $60 \%$ des AOTU. Cette évolution oblige désormais ces AOTU à fournir une solution de transport collectif dans les territoires périphériques où les transports collectifs ne sont pas toujours dans leur domaine de pertinence. La fourniture de services de transport collectifs performants dans des territoires façonnés par l'automobile est en effet une tâche complexe (Drevelle, 2015; Drevelle, Emangard, 2015). Dans les espaces périphériques des métropoles, le radioconcentrisme et l'axialité des déplacements, qui favorisaient les transports collectifs classiques ont diminué en raison du processus de périurbanisation (Ascher, 2002; Aguiléra, Mignot, 2002). Désormais, les déplacements sont de plus en plus multi-polarisés dans ces territoires (Aguiléra et al., 2017). Or, les réseaux de transports collectifs sont conçus selon une logique de regroupement des voyageurs et fonctionnent de ce fait selon une organisation en lignes et points d'arrêts (stations, gares) qui sont fixes et dont la répartition spatiale est restreinte, afin de fournir un service à coût raisonnable. Dans ce contexte, si dans les zones denses l'offre de transport collectif conserve toute sa légitimité, le constat est différent dans les espaces peu denses à l'urbanisation diffuse où l'enjeu est de promouvoir des transports publics nouveaux, performants pour des déplacements épars dans le temps et dans l'espace.

Le covoiturage apparaît comme une réponse possible à la dispersion des déplacements et à l'individualisation des modes de vie. À la différence des transports collectifs, le covoiturage peut potentiellement offrir une grande diversité de trajets et de lieux de prise en charge. Il permet ainsi en théorie de répondre de manière plus fine aux besoins qu'une approche par l'offre de transport public.

Dans ce contexte, depuis 2012 environ, les collectivités territoriales ont commencé à intégrer dans les cahiers des charges des appels d'offres des Délégations de Service Public auxquels les entreprises de transports collectifs répondent afin d'obtenir les marchés locaux de transport public. Le covoiturage est encore cependant dans la grande majorité des cas présenté sous la forme d'une option à lever et non pas comme un élément central de la prestation attendue du transporteur. En ce sens, le covoiturage n'est pas déterminant pour l'attribution des marchés. Toutefois, les transporteurs indiquent s'en saisir de manière stratégique, en positionnant les services de covoiturage comme des compléments de leur offre traditionnelle, en particulier dans les espaces périphériques. Dans ce contexte, les prestations des transporteurs en matière de covoiturage s'étoffent et ils proposent désormais un éventail de services de plus en plus variés aux collectivités.

Seulement, le covoiturage restant une option dans les cahiers des charges, les prestations en la matière ne sont de ce fait pas déterminantes dans l'attribution du mandat de délégataire. Pour les transporteurs, ces services sont donc essentiellement un moyen de se démarquer de leurs concurrents autrement que par les prix.

« On n'est pas arrivé aujourd'hui au stade où c'est le covoiturage qui fait pencher la

balance. Parce que le covoiturage reste accessoire, c'est la cerise sur le gâteau. Si ça 
n'y est pas, c'est un problème, mais si ça y est, c'est considéré comme normal. »

(Transporteur $n^{\circ} 1,2016$ ) n'est pas, selon eux, dans son domaine de pertinence. Les solutions de covoiturage ne sont de ce fait jamais envisagées comme pouvant être déployées de façon totalement indépendante des réseaux de bus. Ces transporteurs présentent donc le covoiturage auprès des collectivités comme un service complémentaire, qui ne peut se passer d'un investissement conséquent sur l'offre de transport collectif traditionnel.

" Même si j'étais convaincu que le covoiturage ce serait beaucoup mieux qu'un bus, vous aurez du mal à me faire enlever un bus pour mettre une station de covoiturage à la place. Je ne vais pas me tirer une balle dans le pied. Je n'utiliserai le covoiturage que pour gagner un marché chez le concurrent, pas pour baisser mon propre chiffre d'affaires. » (Transporteur $\left.n^{\circ} 3,2016\right)$

En définitive, le covoiturage est saisi par les transporteurs pour remporter des marchés, mais il ne revêt toutefois pour eux qu'un enjeu assez marginal. Ces acteurs consentent à des investissements limités sur le sujet et ils se saisissent du covoiturage pour reconcentrer leur offre de service de bus, mais pas dans une optique de réduction globale de la flotte automobile et des effets de congestion. 


\section{Conclusion} covoiturage. Une première période a été caractérisée par le soutien législatif aux initiatives de covoiturage portées par des acteurs territoriaux qui ont elles-mêmes soutenu une démographie d'entreprises digitales favorisant le covoiturage courte distance. Cela s'est traduit par une concurrence non régulée entre ces initiatives ayant conduit à une absence de visibilité de l'offre particulièrement contreproductive pour l'adoption de la pratique. Dans un second temps, les transporteurs se sont saisis du covoiturage pour se démarquer et affirmer leur position, remporter des marchés tandis que les startups se sont divisées en deux groupes assez nettement distincts, celles prônant une libéralisation du secteur et celles prônant l'intégration du covoiturage dans le service public. Parallèlement, l'intérêt des collectivités territoriales sur le sujet s'est renforcé et deux questions se posent. La première concerne la redéfinition de l'activité de covoiturage, entre pratique solidaire ou objet d'action publique reposant sur un modèle d'affaire. Dans ce cas, plutôt que de fonder le covoiturage sur la notion de partage de frais entre covoitureur et covoituré, l'inscription de cette pratique dans l'action publique induit l'idée que le conducteur fasse des profits, qu'il soit intéressé à offrir ses sièges libres aux passagers, et que cette valeur soit mobilisée par des circuits d'acteurs à l'interaction entre système public et système privé, dans des dispositifs économiques et juridiques qui sont en voie de clarification. La deuxième question renvoie à la régulation de ce marché entre libéralisation et contrôle par les acteurs locaux.

es transporteurs possèdent bien davantage de ressources et d'expertise, une capacité à intégrer les innovations et à les adapter aux territoires, mais s'ils deviennent des acteurs pivots dans le système naissant du covoiturage, il existe aussi un risque qu'ils freinent son développement. De leur côté, les startups de covoiturage peuvent aussi développer des systèmes qui entrent directement en concurrence avec les services publics de transport collectifs, et ce d'autant plus si la collectivité subventionne les usagers du covoiturage.

En 2019, la promulgation de la LOM (Loi d'orientation des mobilités) accorde une part centrale au covoiturage dans les politiques publiques de mobilité et tente de créer un cadre organisationnel entre acteurs privés d'une part, entre acteurs privés et publics. Elle entérine la déconnexion du produit covoiturage de la notion de partage de frais, en autorisant les autorités locales et les entreprises à subventionner les usagers qui covoiturent, ce qui, à terme, peut conduire à des bouleversements importants dans la définition et dans l'organisation des services publics de mobilité en France.

47

Plus largement, l'intégration du covoiturage dans les politiques publiques de mobilité soulève l'enjeu du renforcement de l'usage de l'automobile dans la mobilité quotidienne. Or, au regard des relations qu'entretiennent un territoire et son système de mobilité (Saint Amand, 2010), cette dynamique peut avoir des conséquences sur la structuration et l'organisation spatiale des territoires. Plusieurs études alertent déjà sur les externalités négatives liées au covoiturage. Parce que le covoiturage réduit à la fois le coût (partage des frais) et le temps (via la baisse de la congestion) des déplacements en voiture, il génère divers "effets rebonds " atténuant fortement la baisse espérée de l'usage de la voiture. Par ailleurs, en réduisant le coût de se déplacer en voiture, le covoiturage peut inciter certains ménages à résider plus loin des centres 
d'emploi pour pouvoir bénéficier de prix immobiliers plus faibles. En définitive, le développement du covoiturage pourrait ainsi renforcer les phénomènes d'étalement urbain et de périurbanisation, et par conséquent la dépendance automobile de ménages le plus souvent modestes, qui ne trouvent à se loger qu'en périphérie des centres d'emplois (Yin et al., 2017 ; Coulombel et al., 2019). En outre, le fait que le covoiturage puisse permette de diversifier l'offre de déplacement est questionnable. Les services contribueraient à surtout renforcer l'accessibilité des principales polarités des territoires qui sont déjà bien desservis en transports publics (Castex, 2017). Enfin, un service public du covoiturage amorce une potentielle transition où l'usager ne consomme plus un service produit par la puissance publique, mais participe directement à sa production (Lulin, 2013). Cette situation peut se traduire par des phénomènes de discriminations sociales et par des inégalités territoriales auxquelles il importe de prêter une attention particulière (Delaunay, 2018).

\section{BIBLIOGRAPHIE}

ADEME (2017), Leviers d'actions pour favoriser le covoiturage de courte distance, évaluation de l'impact sur les polluants atmosphériques et le $\mathrm{CO}^{2}$, Rapport réalisé par N. Mercat et $\mathrm{P}$. Sucche (Inddigo) pour le compte de l'ADEME, $133 \mathrm{p}$.

AGUILERA A., MIGNOT D. (2002), Formes urbaines et mobilité, Actes du VIII ${ }^{\mathrm{e}}$ Colloque de l'ASRDLF, Trois-Rivières, 21-23 août 2002, 24 p.

AGUILÉRA A., CONTI B. \& LE NÉCHET F. (2017), « Accompagner la transition vers des mobilités plus durables dans le périurbain ", Transports Urbains, 13, février, pp. 3-9.

AKRICH M., CALLON M. \& LATOUR B. (1988), «A quoi tient le succès des innovations ? 1 : L'art de l'intéressement. $2:$ Le choix des porte-parole ", Gérer et Comprendre. Annales des Mines, Les Annales des Mines, pp. 4-17 \& 14-29, 0.1016/j.trd.2018.12.006.

ALTER N. (2003), L'innovation ordinaire, Paris, PUF.

BARTHES A. (2009), Typologie des covoitureurs : analyse de quelques déterminants de passage à l'acte, Club Innovations-transports des Collectivités, CERTU, Ministère du développement durable.

BULTEAU J., FEUILLET T. \& DANTAN S. (2019), “Carpooling and carsharing for commuting in the Paris region: A comprehensive exploration of the individual and contextual correlates of their uses", Travel Behaviour and Society, 16, pp. 77-87.

CASTEX E. (2017), « Le covoiturage, entre concurrence et complémentarité avec les transports en commun : quelle accessibilité à la ville ? Application à l'Aire métropolitaine Lilloise ", in NEGRONPLOBETE P., PAUILLAC F. (dir.), Villes à vivre : le quotidien métropolitain entre ancrage et mobilité, Presses Universitaires de Laval, pp. 175-192.

CGDD (2016), « Covoiturage longue distance : état des lieux et potentiel de croissance », Études et documents, 146.

CGDD (2018), Les facteurs d'évolution des émissions de $\mathrm{CO}_{2}$ liées à l'énergie en France entre 1990 et 2016, Datalab, $38 \mathrm{p}$. 
CLAVEL R., LEGRAND P. (2009), Le covoiturage dynamique : étude préalable avant expérimentation, Lyon, CERTU.

COULOMBEL N., BOUTUEIL V., LIU L., VIGUIÉ V. \& YIN B. (2019), “Substantial rebound effects in urban ridesharing: Simulating travel decisions in Paris, France", Transportation Research Part D: Transport and Environment, 71, https://doi.org/1, pp. 110-126.

CRENO L. (2016), Covoiturer entre inconnus : des risques perçus à la construction de la confiance, panorama des expériences vécues des usagers, Thèse de doctorat en psychologie ergonomique, Télécom ParisTech - ITE Vedecom.

DELAUNAY. T. (2018), L'intégration du covoiturage dans le système de mobilité francilien : hybrider le transport collectif et individuel pour asseoir l'hégémonie de l'automobile?, Thèse de doctorat en architecture, aménagement de l'espace, Université Paris-Est.

DESPORTES M. (2005), Paysages en mouvement. Perception de l'espace et transports (XVIII ${ }^{e}-X X^{e}$ siècle), Gallimard, coll. Bibliothèque des Histoires.

DREVELLE M. (2015), Desservir les faibles densités par les transports collectifs routiers : des réseaux aux prises avec le territoire, Thèse de doctorat en géographie, Université Paris 1-Panthéon Sorbonne.

DREVELLE M., EMANGARD P.H. (2015), Atlas de la France périurbaine, Economica, coll. Méthodes et Approches, $224 \mathrm{p}$.

DUPUY G. (1995), Les territoires de l'automobile, Paris, Economica, coll. Villes.

FAIVRE D'ARCIER B., BRUN G. (2012), Financement durable des transports publics urbains. De la prospective à l'aide à la décision, Le Point sur (Commissariat Général au Développement durable), $140 \mathrm{p}$.

FERRET A., DEMOLY E. (2019), « Les comportements de consommation en 2017. Le transport pèse plus en milieu rural, le logement en milieu urbain », Insee Première, 1749, paru le 17/04/2019.

JULIEN B. (2016), Usages novateurs de la voiture et nouvelles mobilités, Rapport prospective MEDDE, janvier.

LEFÈVRE C., OFFNER J.M. (1990), Les transports urbains en question : Usages - Décisions Territoires, CELSE.

LEJOUX P. (2014), « Les entreprises face à la mutation énergétique : l'enjeu de la mobilité dans les territoires périurbains de la métropole lyonnaise ", Revue Géographique de l'Est, Mutations des systèmes productifs en France, 54, 1-2.

RICHER C. (2017), « Le financement des transports collectifs à l'heure de la mobilité durable : quel avenir pour le versement transport? ", Métropolitiques, 20 novembre 2017, http:// www.metropolitiques.eu/Le-financement-des-transports-collectifs-a-l-heure-de-la-mobilitedurable-quel.html.

SAJOUS P. (2017), « Mobilité quotidienne automobile en périurbain francilien et changement environnemental : les apports de la notion de savoir-faire », Flux, 107, 1, pp. 3-16.

SHAHEEN S., ADAM COHEN A. (2018), "Shared ride services in North America: definitions, impacts, and the future of pooling", Transport Reviews, doi:10.1080/01441647.2018.1497728.

VINCENT S. (2008), Les altermobilités : analyse sociologique d'usages de déplacements alternatifs à la voiture individuelle. Des pratiques en émergence?, Thèse de doctorat, Université de Paris 5. 
YIN B., LIU L., COULOMBEL N. \& VIGUIÉ V. (2018), “Appraising the environmental benefits of ride-sharing: the Paris region case study”, Journal of Cleaner Production, 177, doi: 10.1016/j.jclepro. 2017.12.186, pp. 888-898.

\section{NOTES}

1. Le versement transport (VT ou taxe-transport) est un impôt affecté aux autorités organisatrices de la mobilité (AOM). Il est prélevé sur la masse salariale acquittée par tous les employeurs publics et privés de plus de 11 salariés situés dans le ressort territorial de l'AOM.

2. En France, la délégation de service public (abrégé en DSP) est une notion juridique qui recouvre l'ensemble des contrats par lesquels une personne morale de droit public soumise au code général des collectivités territoriales confie la gestion d'un service public dont elle a la responsabilité à un opérateur économique dont la rémunération est substantiellement liée au résultat d'exploitation du service.

3. Plusieurs entreprises comme Heetch, Jump, Citygoo proposent ainsi des services qui ne respectent pas le montant kilométrique supérieur au partage des frais qui sont définis par le barème kilométrique et qui tournent autour de 0,30 à $0,40 € / \mathrm{km}$. Ce faisant, ils concurrencent les services de VTC et de taxis. Ces entreprises ont été jugées coupables de coupable de complicité d'exercice illégal de la profession de taxi, de pratique commerciale trompeuse et d'organisation illégale d'un système de mise en relation de clients avec des chauffeurs non professionnels. Elles ont dû modifier la nature de leur service pour continuer à opérer en France.

4. L'idée est de permettre aux usagers de bénéficier des trajets effectués chaque jour par de nombreux automobilistes en donnant aux conducteurs et aux passagers un moyen de s'identifier réciproquement en temps réel, sans réservation préalable, grâce à des arrêts de covoiturage aménagés sur la voirie. L'objectif de ces acteurs du covoiturage est de créer un réseau de points de prise en charge sécurisés et bien identifiés, positionnés le long d'axes suffisamment fréquentés pour générer des appariements spontanés, sécurisés et rapides. Schématiquement, le passager se poste sur un arrêt situé en bordure de voirie, de façon équivalente à un arrêt de bus, et signale aux automobilistes qui circulent devant lui qu'il cherche à atteindre un lieu situé sur le trajet de ces automobilistes. Ces derniers peuvent, s'ils le souhaitent et s'ils se rendent effectivement dans la même direction que le passager, prendre à leur bord ce dernier.

5. La forme planifiée aux actifs automobilistes des espaces diffus, la forme dynamique aux usagers des transports collectifs résidant dans un espace métropolitain, la forme spontanée pour les publics précaires des territoires ruraux.

\section{RÉSUMÉS}

Cet article porte sur les positionnements réciproques des pouvoirs publics et des opérateurs privés de mobilité à propos du covoiturage sur courtes distances. Il étudie les conditions commerciales, organisationnelles et institutionnelles de promotion et d'encadrement de cette pratique. Il apporte des pistes expliquant pourquoi le covoiturage peine à émerger dans les pratiques de déplacement, et présente comment il s'intègre désormais dans l'action publique locale. Il mobilise pour cela les théories de la sociologie de l'innovation et utilise les informations 
extraites d'une quarantaine d'entretiens (partie 1). Il retrace tout d'abord le processus d'intégration du covoiturage dans le champ de l'action publique (partie 2). Puis, nous précisons comment et pourquoi s'amorce une diversification des formes de gouvernance et de promotion du covoiturage à travers, d'une part, l'intégration des opérateurs de covoiturage dans le champ du service public de transports (partie 3), et d'autre part, à travers l'implication croissante des entreprises historiques de transports collectifs urbains sur ce marché (partie 4). L'article montre qu'en fonction du type d'acteurs impliqués, cette transition augure un potentiel renforcement de la place du covoiturage dans les services publics de mobilité, mais peut aussi se traduire par une contention de ce mode dans les marges de l'offre de mobilité. Pour conclure, il montre dans quelle mesure ces évolutions soulèvent de nombreux enjeux en termes de mise en œuvre et de régulation, et posent également question quant au renforcement de la place de l'automobile dans le système de mobilité.

This paper deals with the varied governance frameworks established in France between local government and private mobility operators regarding short distance ridesharing services. It focuses on the private operator's business operation and public policies implemented by local governments in order to promote and regulate carpooling. It gives insight into why emergence of carpooling faces difficulty in suburban areas, and presents its current process of integration into local mobility public policies. The theoretical framework is based upon innovation's sociology and our data are issued from forties semi-structured interviews (section 1). First, we trace back the integration process of carpooling into local public policies in France (section 2). Then, we specify how and why a diversification of governance's forms of carpooling is being initiated through, on the one hand, the integration of ridesharing operators into the scope of public transport services (part 3), and on the other hand, through the growing involvement of historic public transport companies into this market (part 4). This paper shows that, depending on the type of actors involved, this transition augurs a potential strengthening of the place of carpooling in public mobility services, but can also result in a restraint of this mode in the margins of the mobility offer. To conclude, it shows to what extent these developments raise many challenges in terms of implementation and regulation, and also raises questions about strengthening the role of the automobile in the mobility system.

\section{INDEX}

Keywords : carpooling, ridesharing, public transport, innovation, governance, France

Mots-clés : covoiturage, mobilité partagée, transports publics, innovation, gouvernance, France

\section{AUTEURS}

\section{TEDDY DELAUNAY}

Laboratoire Ville Mobilité Transport (Université Paris-Est Marne-la-Vallée ; Ecole Nationale des

Ponts ParisTech ; IFSTTAR), teddy.delaunay@enpc.fr

\section{NACIMA BARON}

Laboratoire Ville Mobilité Transport (Université Paris-Est Marne-la-Vallée ; Ecole Nationale des Ponts ParisTech ; IFSTTAR), nacimabaron@gmail.com 